\title{
Clinically acceptable values of shear bond strength of orthodontic brackets bonded on
}

\section{enamel: An integrative review}

Valores clinicamente aceitáveis de resistência ao cisalhamento de bráquetes ortodônticos colados ao

esmalte: Uma revisão integrativa

Valores clínicamente aceptables de resistencia al cizallamiento de los soportes ortodóncicos

adheridos al esmalte: Una revisión integradora

Received: 03/15/2021 | Reviewed: 03/21/2021 | Accept: 03/24/2021 | Published: 04/01/2021

Itanielly Dantas Silveira Cruz

ORCID: https://orcid.org/0000-0003-3271-4588

Federal University of Rio Grande do Norte, Brazil

E-mail: itanielly.cruz@hotmail.com

Amanda Felix Gonçalves Tomaz

ORCID: https://orcid.org/0000-0001-6083-7345

Federal University of Rio Grande do Norte, Brazil

E-mail: amandafelixgoncalves@gmail.com

Mariana Cabral Moreno

ORCID: https://orcid.org/0000-0001-6801-4677

Federal University of Rio Grande do Norte, Brazil

E-mail: marianacabralmoreno@hotmail.com

Rafaela Monteiro de Araújo

ORCID: https://orcid.org/0000-0001-6439-2830

Federal University of Rio Grande do Norte, Brazil

E-mail: rafaelamont@hotmail.com

Arthur César de Medeiros Alves

ORCID: https://orcid.org/0000-0002-4282-4142

Federal University of Rio Grande do Norte, Brazil

E-mail: arthurcesar_88@hotmail.com

Hallissa Simplício Gomes Pereira

ORCID: https://orcid.org/0000-0001-5398-6318

Federal University of Rio Grande do Norte, Brazil

E-mail: hallissa@hotmail.com

Sergei Godeiro Fernandes Rabelo Caldas

ORCID: https://orcid.org/0000-0002-1684-5169

Federal University of Rio Grande do Norte, Brazil

E-mail: sergeirabelo@icloud.com

\begin{abstract}
Objective: The objective this review was to identify the current range of shear bond strength values (SBS) that are clinically acceptable based on a synthesis of the studies published in 20 years that evaluated the SBS of brackets bonded to dental enamel with emitter diode light photo activators and whether damage to the enamel has been reported after the test. Methodology: A search strategy was developed for the selection of articles published from 1999 to 2019 in the PubMed/MedLine electronic database. In addition to reading the titles, keywords and summaries of the studies found, the studies were also obtained for the reading of the methodologies and identification of the inclusion and exclusion criteria. Results: The search for scientific articles resulted in 580 studies, but only 14 were selected based on the preestablished criteria. Ten presented methodological quality to be included in this integrative review, and the mean SBS found was $14,05 \mathrm{MPa}$ with a standard deviation of $\pm 6,52 \mathrm{MPa}$ (range from 7,53 to 20,57 MPa). Conclusion: In conclusion, it was observed that the range of shear bond strength values (5,9 to 7,8 MPa) and taken as a parameter by most authors until now is outdated, since higher values of SBS are feasible without, however, causing dental enamel damage after the debonding of the bracket.
\end{abstract}

Keywords: Shear strength; Dental enamel; Orthodontic brackets.

\section{Resumo}

Objetivo: O objetivo desta revisão foi identificar a faixa atual dos valores de resistência ao cisalhamento (RUC) clinicamente aceitáveis com base em uma síntese dos estudos publicados em 20 anos que avaliaram o RUC de bráquetes colados ao esmalte dentário com fotoativadores de diodo emissor de luz e se foi relatada a presença de danos ao esmalte após o ensaio. Metodologia: Foi desenvolvida uma estratégia de busca para a seleção dos artigos publicados de 1999 a 
2019 na base de dados eletrônica PubMed/MedLine. Além da leitura dos títulos, palavras-chave e resumos dos estudos encontrados, os estudos também foram obtidos para a leitura das metodologias e identificação dos critérios de inclusão e exclusão. Resultados: A busca dos artigos científicos resultou em 580 estudos, mas apenas 14 foram selecionados com base nos critérios pré-estabelecidos. Dez apresentaram qualidade metodológica para serem incluídos nesta revisão integrativa, e a média de RUC encontrada foi de 14,05 MPa com desvio padrão de $\pm 6,52 \mathrm{MPa}$ (intervalo de 7,53 a 20,57 MPa). Conclusão: Em conclusão, observou-se que a faixa de valores de resistência ao cisalhamento (5,9 a 7,8 MPa) tida como parâmetro pela maioria dos autores até o momento está desatualizada, visto que maiores valores de RUC são factíveis sem, no entanto, causar dano ao esmalte dentário após a descolagem do bráquete.

Palavras-chave: Resistência ao cisalhamento; Esmalte dentário; Braquetes ortodônticos.

\section{Resumen}

Objetivo: El objetivo de esta revisión fue identificar el rango actual de valores de resistencia al cizallamiento (RC) que son clínicamente aceptables en base a una síntesis de los estudios publicados en 20 años que evaluaron el RC de soportes adheridos al esmalte dental con diodo emisor foto activadores de luz y si se ha informado daño al esmalte después de la prueba. Metodología: Se desarrolló una estrategia de búsqueda para la selección de artículos publicados desde 1999 hasta 2019 en la base de datos electrónica PubMed/MedLine. Además de la lectura de los títulos, palabras clave y resúmenes de los estudios encontrados, los estudios también se obtuvieron para la lectura de las metodologías e identificación de los criterios de inclusión y exclusión. Resultados: La búsqueda de artículos científicos arrojó 580 estudios, pero solo 14 fueron seleccionados en base a los criterios preestablecidos. Diez presentaron calidad metodológica para ser incluidos en esta revisión integradora, y el RC medio encontrado fue de 14,05 MPa con una desviación estándar de \pm 6,52 $\mathrm{MPa}$ (rango de 7,53 a 20,57 MPa). Conclusión: En conclusión, se observó que el rango de valores de resistencia al cizallamiento $(5,9$ a 7,8 $\mathrm{MPa})$ y tomado como parámetro por la mayoría de los autores hasta ahora está desactualizado, ya que los valores más altos de RC son factibles sin, sin embargo, causando daño al esmalte dental después del desprendimiento del soporte.

Palabras clave: Resistencia al corte; Esmalte dental; Soportes ortodóncicos.

\section{Introduction}

The clinical procedure performed to fix the bracket on the tooth enamel surface is done through the application of polymerizable composite resin at its base, with or without the use of adhesive systems, and may be assisted by photoactivating apparatus. The photoactivation, recommended by Tavas and Watts (1979), is the primordial step of bonding when the photopolymerizable resin is used and is necessary to be able to convert the monomers of the resinous compound into polymer chains, process that is described as degree of conversion and that guarantees the maintenance of the physical, chemical, biological and aesthetic properties of resins (Amato et al., 2014).

The light sources used in this phase may be from light emitting diode (LED), argon laser, halogen light or xenon plasma arc. Proposed by Mills, Jandt and Asworth (1999), LED appliances are the most widely used today and their main differentials relate to no need for cooling fans, the shock resistance, to the silence mode how they work, by generating heat minimum, for having a higher power and a lifetime of 10 thousand hours, on average (Ulusoy et al., 2008).

Like practically everything that is used in contemporary dentistry, the photo activators are also under constant technological evolution. Since the energy density is the result of the multiplication of the irradiation time versus the light intensity $\left(\mathrm{mW} / \mathrm{cm}^{2}\right)$, some authors proposed to reduce the time of photoactivation of the devices, provided that their power was raised to maintain the degree of conversion of the resins inside of the appropriate standards (Rueggeberg, 2011).

In this way, offering a shorter chair time for the patient and a greater practicality for the professional, the new devices launched are presenting a greater power with smaller times for the photoactivation (Mavropoulos. et al., 2008). In this context, the mechanical shear bond test is one of the most established methods in the literature to evaluate the fixation of the bracket to the tooth, and it is imperative - according to Reynolds (1975) - that the appropriate shear bond strength (SBS) is 5,9 to 7,8 MPa. It is known that such a value should not be too low, as would result in the bracket taking off during treatment, and not extremely high, since the removal of it could cause irreversible damage to the dental enamel, such as cracks and/or fractures (Rüger et al., 2011). 
However, this study (Reynolds, 1975) - taken as a parameter by most of researchers - was carried out more than 40 years ago. At that time, the photo activators used were halogen light, and several works performed after the Reynolds obtained values of SBS above the range (5,9 to 7,8 MPa) without causing any damage to the tooth enamel. The aim of this study was to identify the current range of shear bond strength values that are clinically acceptable based on a synthesis of the studies published in the last 20 years that evaluated the SBS of brackets bonded in dental enamel with LED photo activators.

\section{Methodology}

\section{Eligibility criteria:}

The research question of the present review was: "What is the current range of shear bond strength values that can be clinically accepted?"; considering the assistance of the tool designated by the acronym PICOS (Raich e Skelly, 2013), where "P" corresponds to the population, "I" to the intervention, "C" to the comparison/control, "O" to the outcome and "S" to type of study; being:

(P) Population - Orthodontic brackets bonded on dental enamel with LED photo activators;

(I) Intervention - Mechanical shear test;

(C) Comparison/control - Not applicable;

(O) Outcome - Shear bond strength;

(S) Type of study - "In vitro" studies.

The inclusion criteria applied during this stage were: (1) "in vitro" studies, that performed (2) a shear bond test; (3) bonding direct on (4) healthy dental enamel; and (5) evaluation of Adhesive Remanescent Index (ARI) after bracket debonding; and used (6) bovine or human teeth, (7) metal or ceramic brackets and (8) LED photo activator.

The exclusion criteria were: (1) not to mention the load cell used in the shear bond test, (2) the brand or the type of photoactivating device used and (3) the area of the base of the bracket, necessary for the calculation of the SBS in Megapascal (MPa); articles that made (4) the bonding of the bracket on porcelain, dentine or ceramic surface, or on (5) dental enamel that underwent laser therapy/radiotherapy.

\section{Search strategy:}

The search for articles for the bibliographic survey was carried out in the electronic database PubMed/MedLine (https://www.ncbi.nlm.nih.gov/pubmed) including the search strategy: "((orthodontics) AND bracket) AND "shear strength"'".

In addition, these filters were applied: "Abstract" in "Text Availability", "Dental journals" in "Journal", the "English", "Spanish" and "Portuguese" in "Languages" and the time period "from 1999/1/1 to 2019/2/28" in "Publication Date". The start of this period is justified by the year of publication of the paper by Mills, Jandt and Asworth (1999), when they proposed the use of LED photo activators.

Figure 1 shows the flowchart of the data used for the selection of the studies. 
Figure 1 - Flowchart of the data used for the selection of studies.

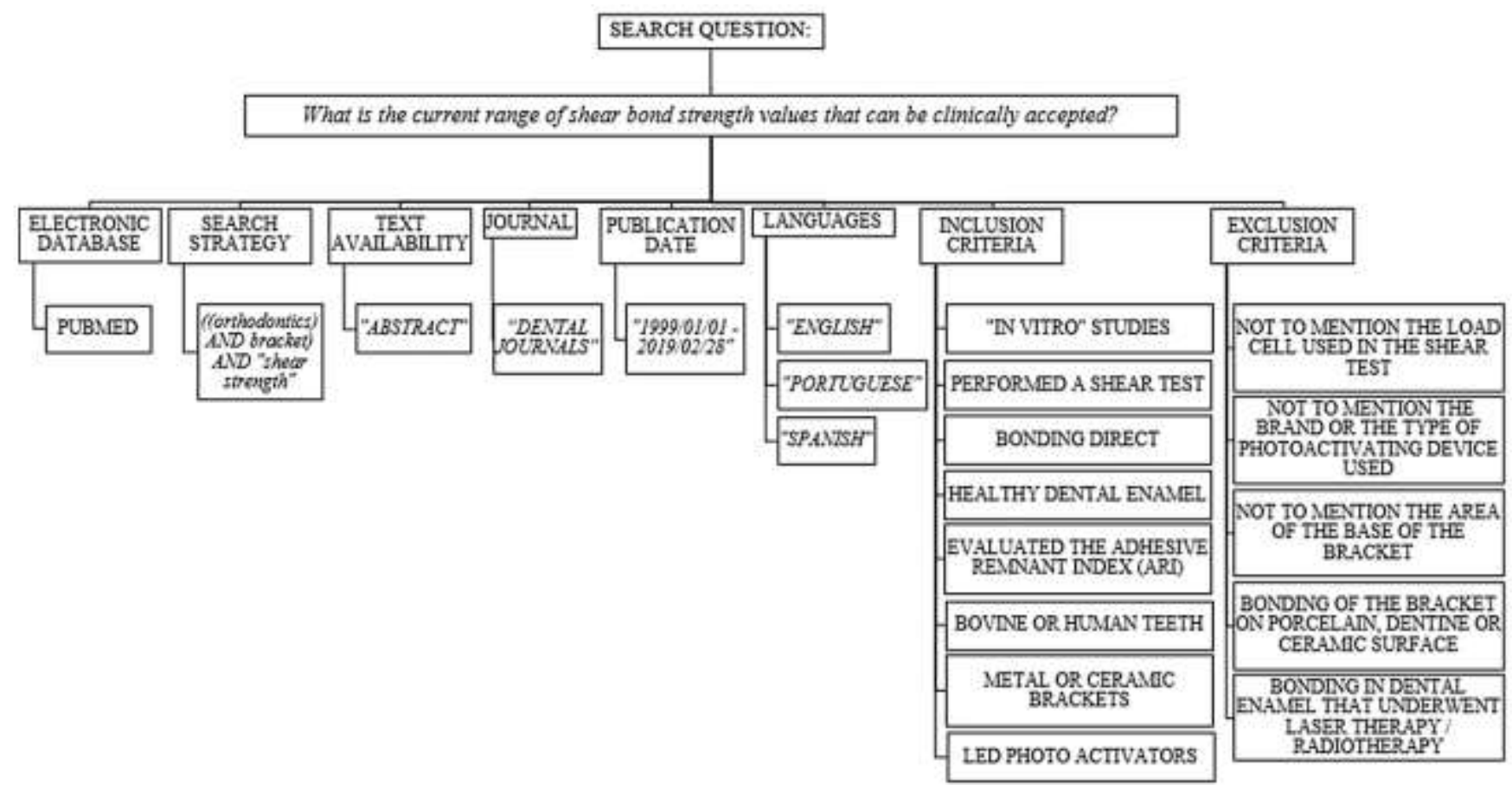

Source: Authors.

\section{Study selection:}

Reading the titles, keywords, abstracts and methodologies of all articles identified was performed independently by two researchers (\#\#\# and \#\#\#) to select those that are relevant to the present study according to the inclusion and exclusion criteria described.

Considering the evidence of the inclusion and exclusion criteria, all studies pertinent to this review were read in full. The complementation of the bibliographic survey was done manually through the analysis of the bibliographic references of the selected articles.

In case of missing or unclear information relevant to decision making, the authors of the study were contacted via email, at least twice in a weekly interval. The reviewers compared their list of manuscripts in each search step; in case of disagreement, the final decision was done by a discussion and consensus with a $3^{\circ}$ reviewer (\#\#\#).

\section{Data extraction:}

Data of interest from included studies were independently extracted and registered by 2 reviewers (\#\#\# and \#\#\#) in a spreadsheet - Excel format by Microsoft Excel ${ }^{\circledR}$ (Microsoft $365^{\circledR}$, Microsoft Corporation, Redmond, WA, EUA).

In addition to the characteristics of the samples of each study, data were also obtained on the following variables: (1) bracket base area, (2) photoactivation protocol, (3) name of the photo activator, (4) load cell used, (5) shear bond strength and (6) whether there was or no damage to the tooth enamel according to the ARI analysis. The principal summary measure was the difference in means of shear bond strengths found in the studies (mentioned in the discussion).

\section{Data analysis:}

The considerable variability of methods and materials from the selected studies made infeasible conducting a metaanalysis. Therefore, a qualitative analysis of results was conducted. 


\section{Results}

Figure 2 corresponds to the flowchart demonstrating the procedures of the article selection strategy and the search result.

Figure 2 - Flowchart demonstrating the procedures of the article selection strategy and the search result.

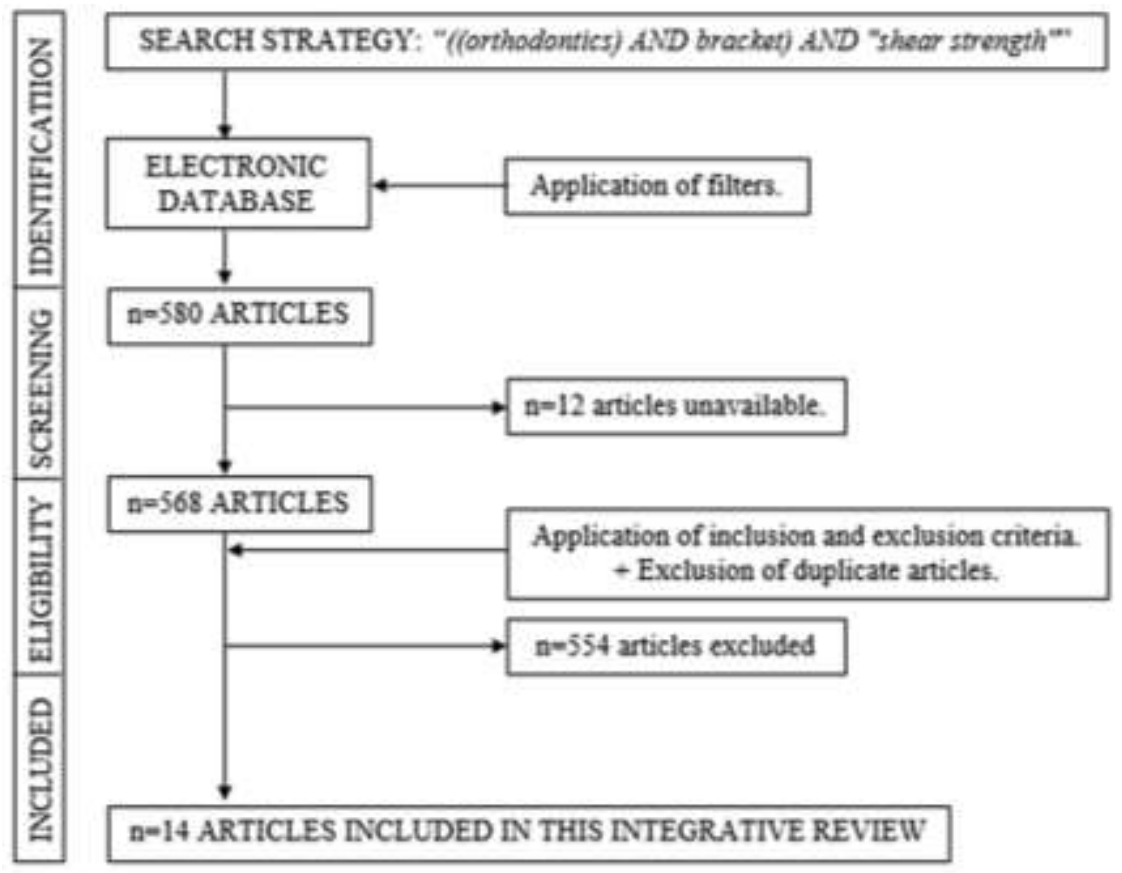

Source: Authors.

The search for scientific articles for the bibliographic survey resulted in 580 studies, but it was not possible to obtain 12 of them (Table 1), even after attempting to contact the authors (by e-mail), and 14 articles (Degrazia et al., 2018; Demirovic et al., 2018; Gupta \& Shrestha, 2018; Ebert et al., 2016; Lee et al., 2016; Tiwari et al., 2016; Mews et al., 2015; Cantekin \& Buyuk, 2014; Kanashiro et al., 2014; Lorenzo et al., 2014; Buyuk et al., 2013; Al-Suleiman, Silikas \& Watts, 2012; Leódido et al., 2012; Namura et al., 2010) were included in this review.

Table 2 contains the description of the articles selected. 
Table 1 - Description of articles not obtained, even after attempting to contact the author(s).

1 Bonding to previously bleached teeth.

TITLE AUTHOR(S)

2 Bond strengths of orthodontic brackets to restorative resin composite surfaces.

3 Shear bond strength of different fixed orthodontic retainers.

4 Strength of attachment between band and glass ionomer cement.

5 The influence of dynamic fatigue loading on the separate components of the bracket-cement-enamel system.

6 Comparison of shear bond strength of orthodontic brackets bonded with light emitting diode (LED).

7 In vitro safety evaluation of a new ultrasound power toothbrush.

8 Comparative assessment of different recycling methods of orthodontic brackets for clinical use.

9 A comparison of shear bond strengths among different self-etching primers.

10 Comparison of the shear bond strength of brackets using the led curing light and plasma arc curing light: polymerization time.

11 Effect of water storage on the shear bond strength of a cyanoacrylate adhesive: clinical implications.

12 Evaluation of a new nano-filled restorative material for bonding orthodontic brackets.

\section{Homewood C, Tyas M, Woods M}

Lai PY, Woods MG, Tyas MJ.

\section{Al-Nimri K, Al-Nimri J.}

Dastjerdie EV1, Zarnegar H, Behnaz M, Seifi M.

Algera TJ, Kleverlaan CJ, Prahl-Andersen B,

Feilzer AJ.

Rachala MR, Yelampalli MR.

Sorensen JA, Pham MM, McInnes C. de Oliveira Correia AM, de Souza Matos F, Pilli Jóias R, de Mello Rode S, Cesar PF, Paranhos LR.

\section{PUBLISHED IN}

Aust Orthod J. 2001 Mar;17(1):27-34.

Aust Orthod J. 1999 Apr;15(4):235-45.

Aust Orthod J. 2015 Nov;31(2):178-83.

Aust Orthod J. 2010 Nov;26(2):149-52.

Am J Dent. 2008 Aug;21(4):239-43.

Int J Orthod Milwaukee. 2010 Winter; 21(4):

31-5.

J Clin Dent. 2008;19(1):28-32.

Minerva Stomatol. 2017 Jun;66(3):107-114.

Evans LS, McGrory KR, English JD, Ontivero

Tex Dent J. 2009 Apr;126(4):312-9.

JC, Powers JM, Frey GN, Duke J.

Yu HS, Lee KJ, Jin GC, Baik HS.

World J Orthod. 2007 Summer;8(2):129-35

Ajlouni R, Bishara SE, Oonsombat C.

World J Orthod. 2004 Fall;5(3):250-3.

Bishara SE, Ajlouni R, Soliman MM, Oonsombat C, Laffoon JF, Warren J.

World J Orthod. 2007 Spring;8(1):8-12.

Source: Authors. 
Table 2 - Description of articles analyzed for a systematic review.

\begin{tabular}{|c|c|c|c|c|c|c|c|}
\hline ARTICLE & SAMPLE CHARACTERISTICS & $\begin{array}{l}\text { BRACKET } \\
\text { (AREA) }\end{array}$ & $\begin{array}{c}\text { PHOTO- } \\
\text { ACTIVATION } \\
\text { PROTOCOL }\end{array}$ & $\begin{array}{c}\text { PHOTO } \\
\text { ACTIVATOR } \\
\text { DEVICE }\end{array}$ & $\begin{array}{l}\text { LOAD } \\
\text { CELL }\end{array}$ & $\begin{array}{l}\text { SHEAR BOND } \\
\text { STRENGTH } \\
\text { (in MPa) }\end{array}$ & $\begin{array}{c}\text { DAMAGE TO } \\
\text { TOOTH } \\
\text { ENAMEL }\end{array}$ \\
\hline $\begin{array}{l}\text { Namura et al., } \\
2010\end{array}$ & $\begin{array}{l}64 \text { bovine teeth ( } 8 \text { groups; } \mathrm{n}=8 \text { ) } \\
\text { GROUPS: } \\
\text { F1: CCF } * 0.001 \% \\
\text { F2: } \mathrm{CCF}^{*}=0.002 \% \\
\text { F3: CCF* }=0.003 \% \\
\text { GC: Group control } \\
\text { SUBGROUPS: } \\
\text { Immediately tested (I) } \\
\text { Tested } 24 \text { hours after bracket bonded ( } 24) \\
* \text { CCF }=\text { Concentration of fluorescent dye derived } \\
\text { from coumarin in the resin }\end{array}$ & $\begin{array}{l}\text { Metallic }(15,26 \\
\left.\mathrm{mm}^{2}\right)\end{array}$ & $\begin{array}{l}20 \text { seconds ( } 10 \text { in the } \\
\text { mesial and } 10 \text { in the } \\
\text { distal) }\end{array}$ & $\begin{array}{l}\text { Ortholux (3M } \\
\text { Unitek) }\end{array}$ & $\begin{array}{l} \pm 1 \mathrm{kN} \\
(1.000 \mathrm{~N})\end{array}$ & $\begin{array}{l}F 1(I)=7,2 \pm 1,2 \\
\text { F1 }(24)=17,0 \pm 3,6 \\
\text { F2 }(I)=6,6 \pm 1,0 \\
\text { F2 }(24)=16,2 \pm 3,2 \\
\text { F3 }(I)=5,2 \pm 0,6 \\
\text { F3 }(24)=9,3 \pm 2,2 \\
\text { GC }(I)=7,3 \pm 1,2 \\
\text { GC }(24)=18,6 \pm 4,4\end{array}$ & No \\
\hline $\begin{array}{l}\text { Al-Sulei-man, } \\
\text { Silikas e Watts, } \\
2012\end{array}$ & $\begin{array}{l}60 \text { human premolars ( } 5 \text { groups; } \mathrm{n}=12 \text { ) } \\
\text { Group 1: SEP Transbond TM Plus (3M Unitek) for } 5 \\
\text { seconds (group control) } \\
\text { Groups 2 a 5: Excluded }\end{array}$ & $\begin{array}{l}\text { Metallic }(12,40 \\
\left.\mathrm{mm}^{2}\right)\end{array}$ & $\begin{array}{l}40 \text { seconds (no } \\
\text { protocol was cited) }\end{array}$ & $\begin{array}{l}\text { BluePhase LED } \\
\text { (Ivoclar } \\
\text { Vivadent) }\end{array}$ & $500 \mathrm{~N}$ & $\mathrm{G} 1=10,00 \pm 4,48$ & No \\
\hline $\begin{array}{l}\text { Leódido et al., } \\
2012\end{array}$ & $\begin{array}{l}48 \text { bovine teeth ( } 4 \text { groups; } n=12) \\
\text { Group GC: No treatment (control) } \\
\text { Group FN: Application of neutral fluorine for } 4 \text { min } \\
\text { before bonded the bracket } \\
\text { Groups FFA e VFS: Excluded }\end{array}$ & $\begin{array}{l}\text { Metallic }(6 \\
\left.\mathrm{mm}^{2}\right)\end{array}$ & $\begin{array}{l}20 \text { seconds }(10 \text { in the } \\
\text { mesial and } 10 \text { in the } \\
\text { distal) }\end{array}$ & $\begin{array}{l}\text { Optilight } \\
\text { (Gnatus, Ribeirão } \\
\text { Preto, SP, Brasil) }\end{array}$ & $\begin{array}{l}50 \mathrm{Kg}(\cong \\
500 \mathrm{~N})\end{array}$ & $\begin{array}{l}\mathrm{G} \mathrm{GC}=13,80 \pm 1,62 \\
\mathrm{G} \mathrm{FN}=11,42 \pm 1,96\end{array}$ & No \\
\hline Buyuk et al., 2013 & $\begin{array}{l}120 \text { human premolars ( } 2 \text { groups; } n=60) \\
\text { Group 1: Transbond XT ( } 3 \mathrm{M} \text { Unitek) } \\
\text { Group 2: Filtek Silorane (3M ESPE) }\end{array}$ & $\begin{array}{l}\text { Metallic }(12,13 \\
\left.\mathrm{mm}^{2}\right)\end{array}$ & $\begin{array}{l}\text { Group 1: } 40 \text { seconds } \\
\text { (20 in the mesial and } \\
20 \text { in the distal) } \\
\text { Group 2: } 40 \text { seconds } \\
\text { (according to } \\
\text { manufacturer) }\end{array}$ & $\begin{array}{l}\text { Valo (Ultradent } \\
\text { Products, South } \\
\text { Jordan, USA) }\end{array}$ & $\begin{array}{l}50 \mathrm{Kg}(\cong \\
500 \mathrm{~N})\end{array}$ & $\begin{array}{l}\mathrm{G} 1=13,61 \pm 4,68 \\
\mathrm{G} 2=4,53 \pm 2,34\end{array}$ & No \\
\hline $\begin{array}{l}\text { Cantekin e Buyuk, } \\
2014\end{array}$ & $\begin{array}{l}\text { 150 human premolars (5 groups; } \mathrm{n}=30 \text { ) } \\
\text { Group 1: Transbond XT (3M Unitek) } \\
\text { Group 2: Experimental fluid composite (SureFil } \\
\text { SDR Flow). } \\
\text { Group 3: Conventional fluid composite (Filtek } \\
\text { Flow, 3M ESPE) } \\
\text { Group 4: Conventional fluid composite (Grandio } \\
\text { Flow, VOCO) } \\
\text { Group 5: Conventional fluid composite (Tetric } \\
\text { Flow, Ivoclar Vivadent) }\end{array}$ & $\begin{array}{l}\text { Metallic }(12,13 \\
\left.\mathrm{mm}^{2}\right)\end{array}$ & $\begin{array}{l}40 \text { seconds }(20 \text { in the } \\
\text { mesial and } 20 \text { in the } \\
\text { distal) }\end{array}$ & $\begin{array}{l}\text { Valo (Ultradent } \\
\text { Products Inc, } \\
\text { South } \\
\text { Jordan, Utah, } \\
\text { USA) }\end{array}$ & $\begin{array}{l}50 \mathrm{Kg}(\cong \\
500 \mathrm{~N})\end{array}$ & $\begin{array}{l}\mathrm{G} 1=13,61 \pm 4,18 \\
\mathrm{G} 2=6,51 \pm 2,09 \\
\mathrm{G} 3=7,52 \pm 2,83 \\
\mathrm{G} 4=11,93 \pm 1,15 \\
\mathrm{G} 5=12,84 \pm 1,92 \mathrm{Ma}\end{array}$ & No \\
\hline $\begin{array}{l}\text { Kanashi-ro et al., } \\
2014\end{array}$ & 80 bovine teeth ( 4 groups; $n=20$ ) & $\begin{array}{l}\text { Metallic }(7,67 \\
\left.\mathrm{mm}^{2}\right)\end{array}$ & $\begin{array}{l}40 \text { seconds ( } 10 \text { on } \\
\text { each face: mesial, }\end{array}$ & $\begin{array}{l}\text { Flash Lite } 1401 \\
\text { (Discus Dental, }\end{array}$ & $\begin{array}{l}1 \mathrm{kN} \\
(1.000 \mathrm{~N})\end{array}$ & $\begin{array}{l}\mathrm{G} 1=23,7 \pm 5,0 \\
\mathrm{G} 2=25,3 \pm 5,1\end{array}$ & No \\
\hline
\end{tabular}




\begin{tabular}{|c|c|c|c|c|c|c|c|}
\hline ARTICLE & SAMPLE CHARACTERISTICS & $\begin{array}{l}\text { BRACKET } \\
\text { (AREA) }\end{array}$ & $\begin{array}{c}\text { PHOTO- } \\
\text { ACTIVATION } \\
\text { PROTOCOL }\end{array}$ & $\begin{array}{c}\text { PHOTO } \\
\text { ACTIVATOR } \\
\text { DEVICE }\end{array}$ & $\begin{array}{l}\text { LOAD } \\
\text { CELL }\end{array}$ & $\begin{array}{l}\text { SHEAR BOND } \\
\text { STRENGTH } \\
\text { (in MPa) }\end{array}$ & $\begin{array}{c}\text { DAMAGE TO } \\
\text { TOOTH } \\
\text { ENAMEL }\end{array}$ \\
\hline & $\begin{array}{l}\text { Group 1: Bracket base was cleaned with methyl } \\
\text { methacrylate monomer } \\
\text { Group 2: Bracket base was cleaned with acetone } \\
\text { Group 3: Bracket base was cleaned with aluminum } \\
\text { oxide blasting ( } 50 \mu \text { m particles }) \\
\text { Group 4: Bracket base was cleaned with detergent } \\
\text { and toothbrush }\end{array}$ & & $\begin{array}{l}\text { distal, occlusal and } \\
\text { cervical) }\end{array}$ & $\begin{array}{l}\text { Culter City, CA, } \\
\text { USA) }\end{array}$ & & $\begin{array}{l}\mathrm{G} 3=25,6 \pm 3,7 \\
\mathrm{G} 4=25,7 \pm 4,2\end{array}$ & \\
\hline $\begin{array}{l}\text { Lorenzo et al., } \\
2014\end{array}$ & $\begin{array}{l}60 \text { human premolars (6 groups; } \mathrm{n}=10 \text { ) } \\
\text { Group 1: Without irradiation and with acid attack } \\
\text { (control group) } \\
\text { Group 2: Without irradiation and without acid } \\
\text { attack (control group) } \\
\text { Groups } 3 \text { a 6: Excluded }\end{array}$ & $\begin{array}{l}\text { Metallic }(9,15 \\
\left.\mathrm{mm}^{2}\right)\end{array}$ & $\begin{array}{l}20 \text { seconds ( } 10 \text { in the } \\
\text { occlusal and } 10 \text { in } \\
\text { the cervical) }\end{array}$ & $\begin{array}{l}\text { Bluephase G2 } \\
\text { (Ivoclar- } \\
\text { Vivadent, } \\
\text { Schaän, } \\
\text { Liechtenstein) }\end{array}$ & $\begin{array}{l}10 \mathrm{kN} \\
(10.000 \\
\mathrm{N})\end{array}$ & $\begin{array}{l}\mathrm{G} 1=18,60 \pm 5,00 \\
\mathrm{G} 2=6,40 \pm 2,40\end{array}$ & No \\
\hline Mews et al., 2015 & $\begin{array}{l}320 \text { bovine teeth ( } 8 \text { groups; } n=40) \\
\text { Group } 1 \text { (group control): Acid attack and adhesive } \\
\text { Group 2: Acid attack, only } \\
\text { Groups } 3 \text { a 8: Excluded }\end{array}$ & $\begin{array}{l}\text { Metallic }(12,90 \\
\left.\mathrm{mm}^{2}\right)\end{array}$ & $\begin{array}{l}\text { From mesial to distal } \\
\text { for } 10 \text { seconds }\end{array}$ & $\begin{array}{l}\text { Smartlite PS } \\
\text { (Dentsply } \\
\text { DeTrey) }\end{array}$ & $2 \mathrm{~N}$ & $\begin{aligned} \mathrm{G} 1 & =17,9 \\
\mathrm{G} 2 & =18,1\end{aligned}$ & $\begin{array}{l}\text { Defects in } \\
\text { enamel were } \\
\text { found in } \mathrm{G} 1\end{array}$ \\
\hline Ebert et al., 2016 & $\begin{array}{l}29 \text { bovine teeth ( } 2 \text { control groups) } \\
\text { Group } 1(n=15) \text { : Metallic bracket } \\
\text { Group } 2(n=14) \text { : Ceramic bracket } \\
+ \text { Tests on } 6 \text { surfaces made with restorative } \\
\text { materials: Excluded }\end{array}$ & $\begin{array}{l}\text { Metallic }(11,82 \\
\left.\mathrm{mm}^{2}\right) \text { and } \\
\text { ceramic }(11,88 \\
\left.\mathrm{mm}^{2}\right)\end{array}$ & $\begin{array}{l}40 \text { seconds ( } 10 \text { on } \\
\text { each face: mesial, } \\
\text { distal, occlusal and } \\
\text { cervical) }\end{array}$ & $\begin{array}{l}\text { Valo Ortho (Opal } \\
\text { Orthodontics, } \\
\text { South Jordan, } \\
\text { UT, USA) }\end{array}$ & $\begin{array}{l}1 \mathrm{kN} \\
(1.000 \mathrm{~N})\end{array}$ & $\begin{array}{l}\mathrm{G} 1=59,69 \pm 11,68 \\
\mathrm{G} 2=49,60 \pm 12,01\end{array}$ & $\begin{array}{l}\text { The high shear } \\
\text { forces induced } \\
\text { cracks in } 2 \text { of } \\
\text { the } 15 \text { teeth } \\
\text { with ceramic } \\
\text { brackets and in } \\
10 \text { of the } 15 \\
\text { teeth with the } \\
\text { metal brackets }\end{array}$ \\
\hline Lee et al., 2016 & $\begin{array}{l}80 \text { human premolars (8 groups; } \mathrm{n}=10 \text { ) } \\
\text { GROUPS: } \\
\text { Groups 1 e 5: Standard power mode of the photo } \\
\text { activator Valo (Ultradent) } \\
\text { Groups } 2 \text { e 6: High power mode of the photo } \\
\text { activator Valo (Ultradent) } \\
\text { Groups } 3 \text { e 7: Plasma emulation mode of the photo } \\
\text { activator Valo (Ultradent) } \\
\text { Groups } 4 \text { e 8: Photo activator Ortholux Luminous } \\
\text { (3M Unitek) } \\
\text { SUBGROUPS: } \\
\text { Groups 1, 2, 3 e 4: Metallic bracket } \\
\text { Groups 5, 6, } 7 \text { e 8: Ceramic bracket }\end{array}$ & $\begin{array}{l}\text { Metallic }(12,40 \\
\left.\mathrm{mm}^{2}\right) \text { and } \\
\text { ceramic e } \\
\left(11,54 \mathrm{~mm}^{2}\right)\end{array}$ & $\begin{array}{l}\text { In the vestibular } \\
\text { face; times: Groups } 1 \\
\text { e } 5=10 \text { seconds } \\
\text { Groups } 2 \text { e } 6=8 \\
\text { seconds } \\
\text { Groups } 3 \text { e } 7=8 \\
\text { seconds } \\
\text { Groups } 4 \text { e } 8=6 \\
\text { seconds }\end{array}$ & $\begin{array}{l}\text { Valo (Ultradent) } \\
\text { e Ortholux } \\
\text { Luminous }(3 \mathrm{M} \\
\text { Unitek) }\end{array}$ & $500 \mathrm{~N}$ & $\begin{array}{l}\mathrm{G} 1 \cong 13 \\
\mathrm{G} 2 \cong 11 \\
\mathrm{G} 3 \cong 17 \\
\mathrm{G} 4 \cong 11,5 \\
\mathrm{G} 5 \cong 22 \\
\mathrm{G} 6 \cong 22,5 \\
\mathrm{G} 7 \cong 19 \\
\mathrm{G} 8 \cong 27,5\end{array}$ & $\begin{array}{l}\text { It was observed } \\
\text { a fracture in } 6 \\
\text { teeth; being } 1 \\
\text { of the groups } \\
\text { of metal } \\
\text { brackets and } 5 \\
\text { of the groups } \\
\text { of ceramic } \\
\text { brackets }\end{array}$ \\
\hline
\end{tabular}




\begin{tabular}{|c|c|c|c|c|c|c|c|}
\hline ARTICLE & SAMPLE CHARACTERISTICS & $\begin{array}{l}\text { BRACKET } \\
\text { (AREA) }\end{array}$ & $\begin{array}{l}\text { PHOTO- } \\
\text { ACTIVATION } \\
\text { PROTOCOL }\end{array}$ & $\begin{array}{l}\text { PHOTO } \\
\text { ACTIVATOR } \\
\text { DEVICE }\end{array}$ & $\begin{array}{l}\text { LOAD } \\
\text { CELL }\end{array}$ & $\begin{array}{l}\text { SHEAR BOND } \\
\text { STRENGTH } \\
\text { (in MPa) }\end{array}$ & $\begin{array}{l}\text { DAMAGE TO } \\
\text { TOOTH } \\
\text { ENAMEL }\end{array}$ \\
\hline Tiwari et al., 2016 & $\begin{array}{l}60 \text { human premolars ( } 2 \text { groups; } n=30 \text { ) } \\
\text { Group 1: Photoactivation of the resin with the } \\
\text { dental chair light off } \\
\text { Group 2: Photoactivation of resin with with the } \\
\text { dental chair light on. }\end{array}$ & $\begin{array}{l}\text { Metallic }(9,81 \\
\left.\mathrm{mm}^{2}\right)\end{array}$ & $\begin{array}{l}20 \text { seconds (no } \\
\text { protocol was cited) }\end{array}$ & $\begin{array}{l}\text { Did not mention } \\
\text { the brand, only } \\
\text { that it was LED. }\end{array}$ & $500 \mathrm{~N}$ & $\begin{array}{l}\mathrm{G} 1=5,74 \pm 1,13 \\
\mathrm{G} 2=7,71 \pm 1,90\end{array}$ & No \\
\hline $\begin{array}{l}\text { Degrazia et al., } \\
2018\end{array}$ & $\begin{array}{l}60 \text { bovine teeth ( } 3 \text { groups; } \mathrm{n}=15) \\
\text { Three experimental orthodontic resins containing } \\
\text { haloisene nanotubes loaded with Triclosan (TCN- } \\
\text { HNT) in different concentrations: } \\
\text { Group 1 (control group): } 0 \% \\
\text { Group 2: } 5 \% \\
\text { Group 3: } 10 \% \\
\text { Group 4: } 20 \%\end{array}$ & $\begin{array}{l}\text { Metallic }(11,18 \\
\left.\mathrm{mm}^{2}\right)\end{array}$ & $\begin{array}{l}40 \text { seconds (no } \\
\text { protocol was cited) }\end{array}$ & $\begin{array}{l}\text { Radii Cal (SDI, } \\
\text { Bayswater, VIC, } \\
\text { Australia) }\end{array}$ & $500 \mathrm{~N}$ & $\begin{array}{l}\mathrm{G} 1=17,77 \pm 4,70 \\
\mathrm{G} 2=17,23 \pm 4,91 \\
\mathrm{G} 3=13,51 \pm 2,93 \\
\mathrm{G} 4=13,80 \pm 2,22\end{array}$ & No \\
\hline $\begin{array}{l}\text { Demiro-vic et al., } \\
2018\end{array}$ & $\begin{array}{l}60 \text { human premolars ( } 2 \text { groups; } \mathrm{n}=30 \text { ) } \\
\text { Group } 1 \text { (group control): Direct bonding } \\
\text { Group 2: Excluded }\end{array}$ & $\begin{array}{l}\text { Metallic }(14,70 \\
\left.\mathrm{mm}^{2}\right)\end{array}$ & $\begin{array}{l}20 \text { seconds ( } 10 \text { in the } \\
\text { mesial and } 10 \text { in the } \\
\text { distal) }\end{array}$ & $\begin{array}{l}\text { Unilite II (Bien } \\
\text { Air, Bienne, } \\
\text { Suíça) }\end{array}$ & $\begin{array}{l}5 \mathrm{kN} \\
(5.000 \mathrm{~N})\end{array}$ & $\mathrm{G} 1=7,48 \pm 1,61$ & No \\
\hline $\begin{array}{l}\text { Gupta e Shrestha, } \\
2018\end{array}$ & $\begin{array}{l}96 \text { human premolars s (4 groups; } \mathrm{n}=24 \text { ) } \\
\text { Groups } 1 \text { e 2: Excluded } \\
\text { Group 3: LED photo activator for } 5 \text { seconds } \\
\text { Group 4: LED photo activator for } 10 \text { seconds }\end{array}$ & $\begin{array}{l}\text { Metallic }(8,80 \\
\left.\mathrm{mm}^{2}\right)\end{array}$ & $\begin{array}{l}\text { Group } 3: 5 \text { seconds } \\
\text { on the occlusal face } \\
\text { Group } 4: 10 \text { seconds } \\
\text { ( } 5 \text { in the mesial and } 5 \\
\text { in the distal) }\end{array}$ & $\begin{array}{l}\text { Galaxy } \\
\text { (Shanghai Co., } \\
\text { Shanghai, China) }\end{array}$ & $\begin{array}{l}100 \mathrm{kN} \\
(100.000 \\
\mathrm{N})\end{array}$ & $\begin{aligned} \mathrm{G} 3 & =12,49 \pm 4,23 \\
\mathrm{G} 4 & =14,60 \pm 5,12\end{aligned}$ & No \\
\hline
\end{tabular}

Legend: $\mathrm{MPa}=$ Megapascal; $\mathrm{N}=$ Newtons; $\mathrm{kN}=$ Kilonewtons. Source: Authors. 


\section{Discussion}

In 1975, Reynolds (1975) conducted a thorough review on collages in Orthodontics. As a consensus, he concluded that the main causes of bracket collapsing failures are contamination with moisture, and excessive force on orthodontic fittings. In this context, there are several types of mechanical tests of bond strength that simulate what occurs clinically and evaluate the bracket responses to the possible forces that are applied to them when in the mouth so that, later, the techniques and/or materials used during bonding of such orthodontic fittings may be improved (Neto, de Aragão Pedra \& Miguel, 2004).

In orthodontics, the mechanical shear bond test is commonly used test for determining the efficiency of bonding systems (Finnema et al., 2010). According to Millett and McCabe (1996), this type of test is the most reliable to what occurs "in vivo" because the force is applied to the body in the direction perpendicular to its longitudinal axis; that is why the shear bond test was one of the inclusion criteria applied.

The mechanical test is performed in a universal testing machine, to which the devices with the test bodies are adapted. After this device is attached, the rivets, screws or pins are inserted to carry out the force application. During the test, this force will be raised until rupture occurs. When this "cut" occurs, the parts move parallel to each other (by sliding) and separate, this so-called shear phenomenon. As a response to the "shear" stress, the material develops at each point of its cross section a shear bond strength (International Standardization Organization 11405, 1994).

As regards the force measurement that is applied during the mechanical shear test, a load cell coupled to the universal test machine is used, this cells load are force transducers that convert the active load into a measurable electrical output. According to ISO 7500-1 (Associação Brasileira de Normas Técnicas, 2004), the load cell, irrespective of its magnitude, must be calibrated in 05 points corresponding to $20 \%, 40 \%, 60 \%, 80 \%$ and $100 \%$ of its nominal value. However, if the equipment used is for tests with values lower than $20 \%$ of their nominal value, the load cell must be calibrated in another 3 points: $2 \%, 5 \%$ and $10 \%$.

Thus, it is necessary that the strength of the SBS is within the confidence limit (from $2 \%$ to $100 \%$ of the total value of the cell) so that the value found is a reliable data. Of the 14 studies selected for the present review, one (7,14\%) (Mews et al., 2015) used a load cell of 2 Newtons (N); the majority (7; 50\%) (Degrazia et al., 2018; Lee et al., 2016; Tiwari et al., 2016; Cantekin \& Buyuk, 2014; Buyuk et al., 2013; Al-Suleiman, Silikas and Watts, 2012; Leódido et al., 2012) used a 500 N load cell; 21,42\% (3) (Ebert et al., 2016; Kanashiro et al., 2014; Namura et al., 2010) made use of a $1.000 \mathrm{~N}$ load cell; one study $(7,14 \%)$ (Demirovic et al., 2018) used a 5.000 N load cell, other (7,14\%) (Lorenzo et al., 2014) used a $10.000 \mathrm{~N}$ load cell and one study $(7,14 \%)$ (Gupta \& Shrestha, 2018) used a 100.000 N load cell.

The shear bond strength is generated in Newton (N) and converted to Megapascal (MPa) according to the following formula: $\left[S B S(M P a)=F(N) / A\left(\mathrm{~mm}^{2}\right)\right]$, where $\underline{\mathrm{A}}$ is the base area of the bracket. In this sense, considering the load cells used, the areas of the bases of the brackets and the SBS values found in the articles of this review, it is concluded that in 11 studies $(78,57 \%)$ the shear bond strengths obtained are within the confidence limit, but this is not observed in two of them (14,28\%) (Gupta and Shrestha, 2018; Lorenzo et al., 2014) and one study (7,14\%) (Mews et al., 2015) informed of a load cell with a value less than appropriate. Therefore, 3 out of the 14 studies had calibration bias and, consequently, were excluded from the other analyzes of the present review, since it is assumed that they may not be reliable.

According to Reynolds (1975), the shear bond strength appropriate for clinical use should be 5,9 to 7,8 MPa. However, the study of this author was carried out more than 40 years ago, when the photo activators used were only halogen light. Thus, to identify the current range of SBS values that are acceptable to clinical practice, the present review included the use of only LED photo activators, since these are the most used currently, and also the analysis of the Adhesive Remanescent Index to assess whether or not bracket removal has caused irreversible damage to tooth enamel, such as cracks and/or fractures. 
There are several classifications in the literature regarding ARI, but the two commonly used are those proposed by Årtun and Bergland (1984) and Bishara and Trulove (1990). Independently of the classification type, it is ideal that the most observed score in the "in vitro" studies is the one in which the largest amount of resin remains in the enamel, because, in this way, the greater the chances that the resin will also remain on the surface of the tooth in the time of removal of the orthodontic appliance at the end of treatment ("in vivo"), that may, instead of causing irreversible damage to dental enamel, be carefully removed with specific drills.

As can be observed, most of the articles $(9 ; 81,81 \%)$ selected for this review did not report the presence of enamel damage after the mechanical shear test, and the mean SBS found after the analysis of the 11 studies was of 16,14 MPa with standard deviation of $\pm 11,13 \mathrm{MPa}$ (Figure 3 ). However, it is necessary to elucidate that this high standard deviation was due to the results of the article by Ebert et al. (2016), because the authors found shear bond strengths of 49,60 MPa and 59,69 MPa, and these values were classified the outliers in the Box-Plot graph (Figure 4) according to software the Statistical Package for the Social Sciences ${ }^{\circledR}$ (IBM SPSS Statistics, version 20).

Figure 3 - Histogram of SBS values and ARI analysis including 11 studies.

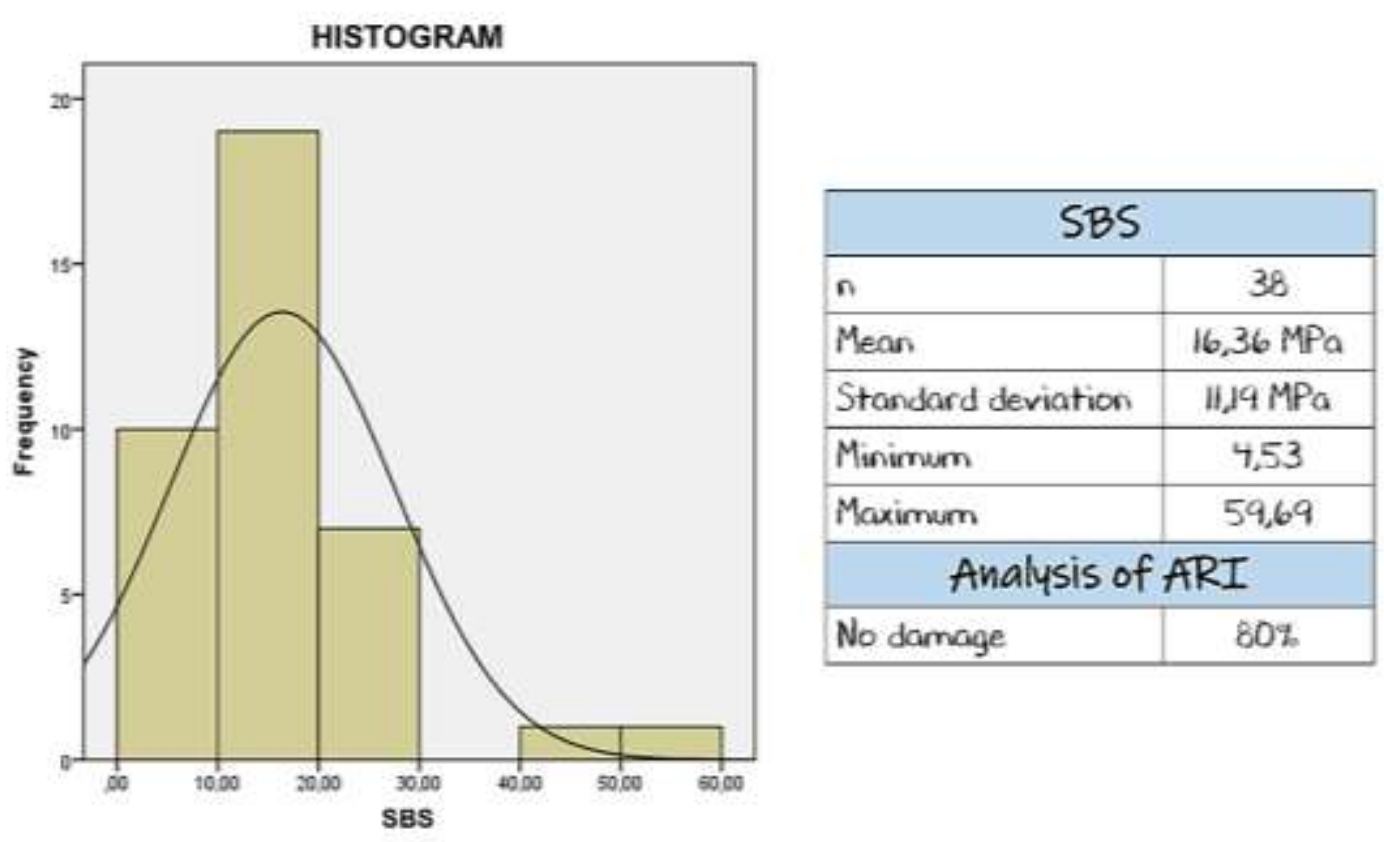

Source: IBM SPSS Statistics 20 and author. 
Figure 4 - Box-Plot graph.

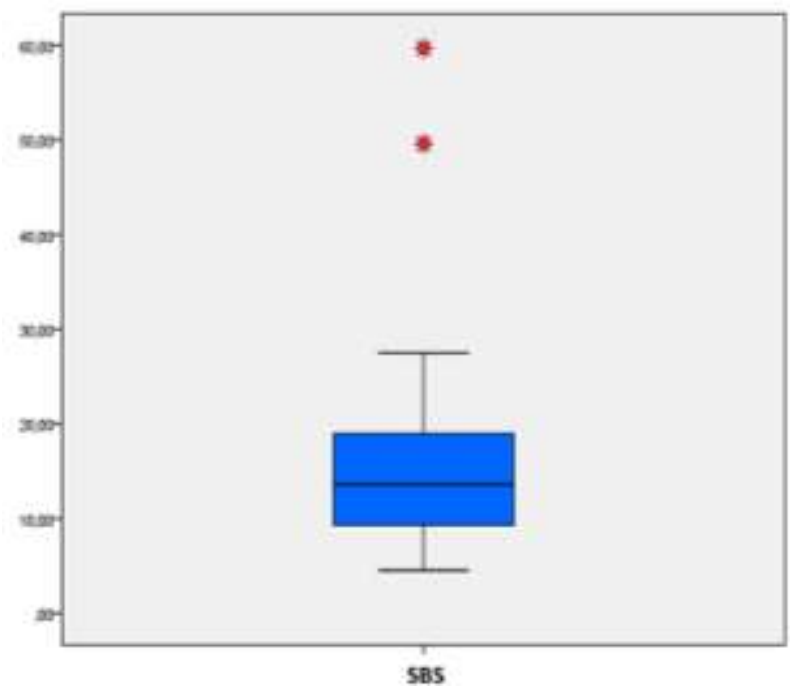

Source: IBM SPSS Statistics 20.

Thus, this study (Ebert et al., 2016) was also excluded from the other analyzes of this review. Thus, there was an increase in the percentage of articles that did not report the presence of damage to the enamel after the shear test from $81,81 \%$ to $90 \%$, and the mean SBS found after the analysis of the 10 studies was 14,05 MPa with standard deviation of \pm 6,52 MPa (Figure 5). This means that most likely the maximum range of SBS values appropriate for clinical use defined by Reynolds in 1975 is outdated and that higher values are feasible without, however, causing enamel damage.

Figure 5 - Histogram of SBS values and ARI analysis including 10 studies.

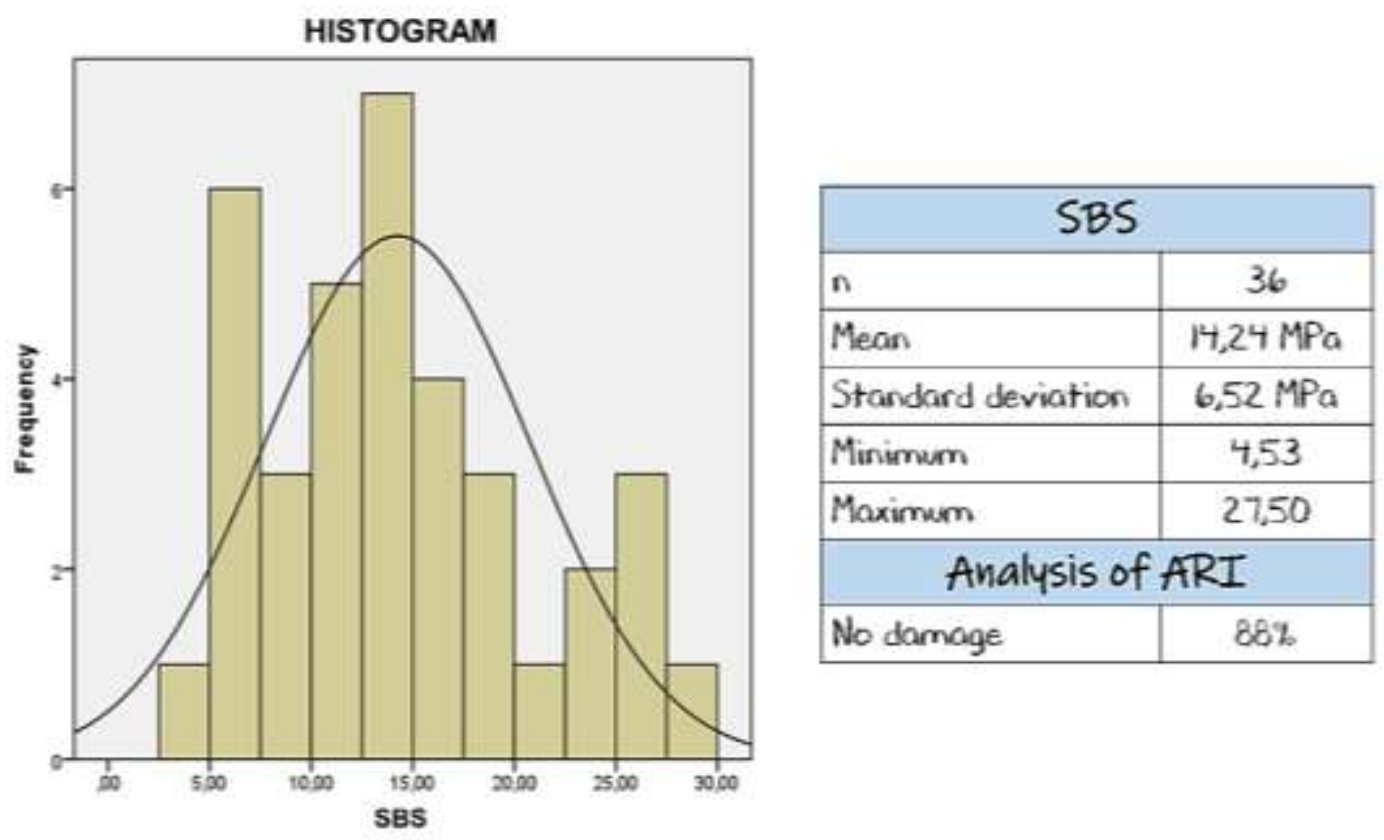

Source: IBM SPSS Statistics 20 and author.

Regarding the articles, although their methodology is similar due to the inclusion criteria applied in this review, some differences should be emphasized, such as the type of resin used and the photoactivation protocol. Some of the studies used 
different resins for the mechanical shear bond test, such as fluid resins (Cantekin \& Buyuk, 2014), of low contraction (Buyuk et al., 2013) and experimental resins containing haloisene nanotubes loaded with Triclosan in different concentrations (Degrazia et al., 2018).

Although not usually used by orthodontists, the groups with these resins presented SBS values above the minimum value recommended by Reynolds (1975), except for the low-contraction resin group (Filtek Silorane®) (Buyuk et al., 2013). The groups of the conventional fluid composites Grandio Flow® (VOCO) and Tetric Flow® (Ivoclar Vivadent), for example, presented SBS values very close to the gold standard resin group, the Transbond XT® (3M Unitek) $(11,93 \pm 1,15 \mathrm{MPa} ; 12,84 \pm$ 1,92 MPa and 13,61 \pm 4,18 MPa, respectively) (Cantekin \& Buyuk, 2014).

Another important factor observed was the photoactivation protocol applied in the articles. In addition to the difference between the photoactivation times (already expected due to the specifications of the device manufacturers), there was also a difference between the photoactivated faces. Excluding the study by Ebert et al. (2016) because it contained outliers, one study (10\%) (Lee et al., 2016) performed the photoactivation only on the vestibular face; one (10\%) (Kanashiro et al., 2014) advocated the photoactivation protocol on the four faces (mesial, distal, cervical and occlusal); 30\% (3) (Degrazia et al., 2018; Tiwari et al., 2016; Al-Suleiman, Silikas \& Watts, 2012) did not mention how the photoactivation was performed and most of the 10 studies (5; 50\%) (Demirovic et al., 2018; Cantekin \& Buyuk, 2014; Buyuk et al., 2013; Leódido et al., 2012; Namura et al., 2010) reported having photoactivated the mesial and distal faces.

Concerning SBS according to the applied protocol, an average ( \pm standard deviation) of $17,93( \pm 5,91) \mathrm{MPa}$ was observed for the photoactivation only on the vestibular face; of 25,07 $( \pm 0,93)$ MPa for the 4 faces and 10,59 ( $\pm 4,29)$ MPa for the photoactivation protocol on the mesial and distal faces. The brackets, by themselves, make the light incidence of the photo activator unfeasible on the whole layer of resin that was inserted in its base by virtue of its opacity. Furthermore, since the degree of conversion depends on several factors, such as the wavelength of light of the photoactivating apparatus, the intensity of the irradiation, the exposure time and the distance between the tip of the photo activator and the resin; it is expected that the conversion of the monomers into polymers will be differentiated depending on the protocol applied, and this will, consequently, interfere with the SBS (Yoshida et al., 2012).

Finally, regarding the limitations and biases found during the development of this review, in addition to the bias verified in 3 of the studies, there was also a lack in the description and methodological quality of articles published in the literature, regardless of the level of the journal, being this the main limitation of this work and of other authors (Finnema et al., 2010). This conclusion was reached because not only the titles, the keywords and the summaries of the articles found after the bibliographic survey were read in full, but also the methodology of all of them. Of the 488 accessed, only 2,86\% (14) could be selected. Many of them did not mention the load cell used, for example, information that is essential when it comes to a mechanical shear test.

\section{Conclusion}

After the results obtained in the present review, it can be concluded that:

- The most used a load cell of $500 \mathrm{~N}$;

- $78.57 \%$ of the studies obtained SBS forces within the confidence limit of the load cell;

- The mean SBS was 14,05 with a standard deviation of $\pm 6,52 \mathrm{MPa}$;

- Regarding ARI, most of the studies did not report the presence of damage to the dental enamel after the mechanical test;

Therefore, the range of shear bond strength values proposed by Reynolds (1975) and taken as a parameter by most authors until now is outdated, since higher values of SBS are feasible without, however, causing dental enamel damage after the debonding of the bracket. 


\section{Acknowledgments}

This research did not receive any specific grant from funding agencies in the public, commercial, or not-for-profit sectors.

\section{References}

Associação Brasileira de Normas Técnicas (2004). Materiais metálicos - máquinas de ensaio estático uniaxial. NBR - ISO 7500-1.

Al-Suleiman, M., Silikas, N., \& Watts, D. (2012). Effects of procedures of remineralization around orthodontics bracket bonded by self-etching primer on its shear bond strength. Journal of orthodontic science, 1(3), 63.

Amato, P. A. F., Martins, R. P., dos Santos Cruz, C. A., Capella, M. V., \& Martins, L. P. (2014). Time reduction of light curing: Influence on conversion degree and microhardness of orthodontic composites. American Journal of Orthodontics and Dentofacial Orthopedics, 146(1), 40-46.

Årtun, J., \& Bergland, S. (1984). Clinical trials with crystal growth conditioning as an alternative to acid-etch enamel pretreatment. American journal of orthodontics, 85(4), 333-340.

Bishara, S. E., \& Trulove, T. S. (1990). Comparisons of different debonding techniques for ceramic brackets: An in vitro study: Part II. Findings and clinical implications. American Journal of Orthodontics and Dentofacial Orthopedics, 98(3), 263-273.

Buyuk, S. K., Cantekin, K., Demirbuga, S., \& Ozturk, M. A. (2013). Are the low-shrinking composites suitable for orthodontic bracket bonding?. European journal of dentistry, 7(3), 284.

Cantekin, K., \& Buyuk, S. K. (2014). Shear bond strength of a new low-shrinkage flowable composite for orthodontic bracket bonding. Journal of Dentistry for Children, 81(2), 63-66.

Degrazia, F. W., Genari, B., Leitune, V. C. B., Arthur, R. A., Luxan, S. A., Samuel, S. M. W., ... \& Sauro, S. (2018). Polymerisation, antibacterial and bioactivity properties of experimental orthodontic adhesives containing triclosan-loaded halloysite nanotubes. Journal of dentistry, $69,77-82$.

Demirovic, K., Slaj, M., Spalj, S., Slaj, M., \& Kobaslija, S. (2018). Comparison of shear bond strength of orthodontic brackets using direct and indirect bonding methods in vitro and in vivo. Acta Informatica Medica, 26(2), 125.

Ebert, T., Elsner, L., Hirschfelder, U., \& Hanke, S. (2016). Shear bond strength of brackets on restorative materials. Journal of Orofacial Orthopedics/Fortschritte der Kieferorthopädie, 77(2), 73-84.

Finnema, K. J., Özcan, M., Post, W. J., Ren, Y., \& Dijkstra, P. U. (2010). In-vitro orthodontic bond strength testing: a systematic review and meta-analysis. American Journal of Orthodontics and Dentofacial Orthopedics, 137(5), 615-622.

Gupta, S. P., \& Shrestha, B. K. (2018). Shear bond strength of a bracket-bonding system cured with a light-emitting diode or halogen-based light-curing unit at various polymerization times. Clinical, cosmetic and investigational dentistry, 10,61 .

International Standardization Organization (1994). Dental Materials: Guidance on testing of adhesion to tooth structure. Geneva, Switzerland: ISO TR 11405.

Kanashiro, L. K., Robles-Ruíz, J. J., Ciamponi, A. L., Medeiros, I. S., Tortamano, A., \& Paiva, J. B. (2014). Influence of different methods of cleaning custom bases on the shear bond strength of indirectly bonded brackets. Journal of orthodontics, 41(3), 175-180.

Lee, H. M., Kim, S. C., Kang, K. H., \& Chang, N. Y. (2016). Comparison of the bonding strengths of second-and third-generation light-emitting diode lightcuring units. Korean journal of orthodontics, 46(6), 364-371.

Leódido, G. D. R., Fernandes, H. O., Tonetto, M. R., Presoto, C. D., Bandéca, M. C., \& Firoozmand, L. M. (2012). Effect of fluoride solutions on the shear bond strength of orthodontic brackets. Brazilian dental journal, 23(6), 698-702.

Lorenzo, M. C., Portillo, M., Moreno, P., Montero, J., Castillo-Oyagüe, R., García, A., \& Albaladejo, A. (2014). In vitro analysis of femtosecond laser as an alternative to acid etching for achieving suitable bond strength of brackets to human enamel. Lasers in medical science, 29(3), 897-905.

Mavropoulos, A., Cattani-Lorente, M., Krejci, I., \& Staudt, C. B. (2008). Kinetics of light-cure bracket bonding: power density vs exposure duration. American journal of orthodontics and dentofacial orthopedics, 134(4), 543-547.

Mews, L., Kern, M., Ciesielski, R., Fischer-Brandies, H., \& Koos, B. (2015). Shear bond strength of orthodontic brackets to enamel after application of a caries infiltrant. The Angle Orthodontist, 85(4), 645-650.

Millett, D. T., \& McCabe, J. F. (1996). Orthodontic bonding with glass ionomer cement. The European Journal of Orthodontics, 18(1), 385-399.

Mills, R. W., Jandt, K. D., \& Ashworth, S. H. (1999). Dental composite depth of cure with halogen and blue light emitting diode technology. British dental journal, 186(8), 388-391.

Namura, Y., Tsuruoka, T., Ryu, C., Kaketani, M., \& Shimizu, N. (2010). Usefulness of orthodontic adhesive-containing fluorescent dye. The European Journal of Orthodontics, 32(6), 620-626.

Neto, C., de Aragão Pedra, J. O., \& Miguel, J. A. M. (2004). Uma análise dos testes in vitro de força de adesão em Ortodontia. Rev. dent. press ortodon. ortopedi. facial, 9(4), 44-51. 
Research, Society and Development, v. 10, n. 4, e11110413927, 2021

(CC BY 4.0) | ISSN 2525-3409 | DOI: http://dx.doi.org/10.33448/rsd-v10i4.13927

Raich, A. L., \& Skelly, A. C. (2013). Asking the right question: specifying your study question. Evidence-based spine-care journal, 4(2), 68-71.

Reynolds, I. R. (1975). A review of direct orthodontic bonding. British journal of orthodontics, 2(3), 171-178.

Rueggeberg, F. A. (2011). State-of-the-art: dental photocuring—a review. Dental materials, 27(1), 39-52.

Rüger, D., Harzer, W., Krisjane, Z., \& Tausche, E. (2011). Shear bond strength after multiple bracket bonding with or without repeated etching. The European Journal of Orthodontics, 33(5), 521-527.

Tavas, M. A., \& Watts, D. C. (1979). Bonding of orthodontic brackets by transillumination of a light activated composite: an in vitro study. British journal of orthodontics, 6(4), 207-208.

Tiwari, A., Shyagali, T., Kohli, S., Joshi, R., Gupta, A., \& Tiwari, R. (2016). Effect of Dental Chair Light on Enamel Bonding of Orthodontic Brackets Using Light Cure Based Adhesive System: An In-Vitro Study. Acta Informatica Medica, 24(5), 317.

Ulusoy, Ç., Irmak, Ö., Bağiş, Y. H., \& Ulusoy, Ö. İ. A. (2008). Temperature rise and shear bond strength of bondable buccal tubes bonded by various light sources. The European Journal of Orthodontics, 30(4), 413-417.

Yoshida, S., Namura, Y., Matsuda, M., Saito, A., \& Shimizu, N. (2012). Influence of light dose on bond strength of orthodontic light-cured adhesives. The European Journal of Orthodontics, 34(4), 493-497. 